\title{
Breakfast consumption and CVD risk factors in European adolescents: the HELENA (Healthy Lifestyle in Europe by Nutrition in Adolescence) Study
}

\author{
Lena Hallström ${ }^{1,2, *}$, Idoia Labayen ${ }^{2,3}$, Jonatan R Ruiz², Emma Patterson², \\ Carine A Vereecken ${ }^{4,5}$, Christina Breidenassel ${ }^{6}$, Frédéric Gottrand 7 , Inge Huybrechts ${ }^{5}$, \\ Yannis Manios ${ }^{8}$, Lorenza Mistura9 ${ }^{9}$ Kurt Widhalm ${ }^{10}$, Katerina Kondaki ${ }^{8}$ \\ Luis A Moreno ${ }^{11}$ and Michael Sjöström², on behalf of the HELENA Study Group \\ ${ }^{1}$ School of Health, Care and Social Welfare, Box 883, Mälardalens University, 72123 Västerås, Sweden: \\ ${ }^{2}$ Unit for Preventive Nutrition, Department of Biosciences and Nutrition, Karolinska Institute, Huddinge, Sweden: \\ ${ }^{3}$ Department of Nutrition and Food Science, University of the Basque Country, Vitoria, Spain: ${ }^{4}$ Research \\ Foundation - Flanders, Brussels, Belgium: ${ }^{5}$ Department of Public Health, Ghent University, Ghent, Belgium: \\ ${ }^{6}$ Institut für Ernährungs- und Lebensmittelwissenschaften-Humanernährung, Rheinische Friedrich-Wilhelms \\ Universität, Bonn, Germany: ${ }^{7}$ Faculté de Médecine, Université de Lille 2, Lille, France: ${ }^{8}$ Department of Nutrition \\ and Dietetics, Harokopio University, Athens, Greece: ${ }^{9}$ National Research Institute on Food and Nutrition, \\ Rome, Italy: ${ }^{10}$ Division of Clinical Nutrition and Prevention, Department of Pediatrics, Medical University \\ of Vienna, Vienna, Austria: ${ }^{1}$ Escuela Universitaria de Ciencias de la Salud, Universidad de Zaragoza, \\ Zaragoza, Spain
}

Submitted 28 February 2011: Final revision received 10 February 2012: Accepted 24 February 2012: First published online 12 April 2012

\begin{abstract}
Objective: To examine the association between breakfast consumption and CVD risk factors in European adolescents.

Design: Cross-sectional. Breakfast consumption was assessed by the statement 'I often skip breakfast' and categorized into 'consumer', 'occasional consumer' and 'skipper'. Blood pressure, weight, height, waist circumference, skinfold thickness, total cholesterol (TC), HDL cholesterol (HDL-C), LDL cholesterol (LDL-C), TAG, insulin and glucose were measured and BMI, TC:HDL-C, LDL-C:HDL-C and homeostasis model assessment-insulin resistance index (HOMA-IR) were calculated. Setting: The European Union-funded HELENA (Healthy Lifestyle in Europe by Nutrition in Adolescence) Study.

Subjects: European adolescents, aged 12.50-17.49 years, from ten cities within the HELENA study ( $n$ 2929, $n 925$ with blood sample, 53\% females).

Results: In males, significant differences across breakfast consumption category ('consumer', 'occasional consumer' and 'skipper') were seen for age, BMI, skinfold thickness, waist circumference, cardiorespiratory fitness, systolic and diastolic blood pressures, TC:HDL-C, LDL-C:HDL-C, glucose, insulin, HOMA-IR and LDL-C; in females, for cardiorespiratory fitness, skinfold thickness, BMI, insulin and HOMA-IR. In overweight/obese males significant differences were also seen for TC and LDL-C, whereas no differences were observed in non-overweight males or in females regardless of weight status.

Conclusions: Our findings among European adolescents confirm previous data indicating that adolescents who regularly consume breakfast have lower body fat content. The results also show that regular breakfast consumption is associated with higher cardiorespiratory fitness in adolescents, and with a healthier cardiovascular profile, especially in males. Eating breakfast regularly may also negate somewhat the effect of excess adiposity on TC and LDL-C, especially in male adolescents.
\end{abstract}

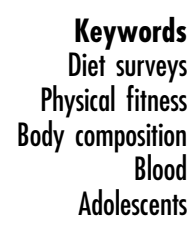

The prevalence of overweight and obesity in adolescence has increased dramatically in developed countries over the past two decades ${ }^{(1)}$. In addition to genetic and environmental factors, the breakfast meal and the frequency with which it is consumed may influence appetite, dietary

intake and composition. These mechanisms may have important implications for body weight regulation. Indeed, several studies have shown a positive association between breakfast skipping and overweight/obesity in adolescents $^{(2-5)}$. 
Breakfast is commonly considered a key component of a healthy diet contributing to whole-diet nutrient adequacy $^{(6,7)}$. Adolescents who rarely have breakfast are more likely to smoke, drink alcohol and are less likely to exercise than regular breakfast consumers ${ }^{(8)}$. Breakfast consumption may reduce the risk of chronic diseases due to its potential impact on overall diet quality ${ }^{(6,9-11)}$. Although breakfast is widely promoted as essential for the nutritional well-being of young people, breakfast skipping is relatively common among adolescents in Western countries ${ }^{(3,12)}$.

Overweight and obesity in childhood are associated with CVD risk factors (adverse levels of lipids, insulin and blood pressure $)^{(13-15)}$. CVD events occur more frequently during or after the fifth decade of life; however, there is evidence indicating that the precursors of CVD have their origin in early childhood ${ }^{(16)}$. Adverse CVD risk factors during childhood have been shown to track later into adulthood $^{(17,18)}$. We hypothesized that if breakfast can be considered a marker of a healthy lifestyle in young people, adolescents who regularly consume breakfast should also have a healthier cardiovascular profile than their peers who skip breakfast.

The purpose of the present study was to examine the association between different patterns of breakfast consumption (skipping, occasional consumption and regular consumption) and CVD risk factors, including BMI, skinfold thickness, waist circumference, cardiorespiratory fitness, blood pressure, blood lipids and insulin resistance, in European adolescents from nine different countries. We also studied the interaction between breakfast consumption and weight status on CVD risk factors.

\section{Methods}

\section{Study design and sampling}

Adolescents were part of the HELENA (Healthy Lifestyle in Europe by Nutrition in Adolescence) Study ${ }^{(19)}$. HELENA is a multi-centre, cross-sectional study performed in ten European cities (Athens and Heraklion in Greece; Dortmund in Germany; Ghent in Belgium; Lille in France; Pecs in Hungary; Rome in Italy; Stockholm in Sweden; Vienna in Austria; Zaragoza in Spain) that was designed to obtain reliable and comparable data on nutrition- and healthrelated parameters of a sample of European adolescents ${ }^{(19)}$.

A total of 3528 ( $52 \%$ females) adolescents, aged 12.50 17.49 years (mean 14.7 (sD) 1.2 years), were recruited between October 2006 and December 2007. Adolescents were randomly selected from schools using proportional cluster sampling taking into account the geographical distribution in each city, the ratio of private to public schools and the number of classes per school. One-third of the classes were randomly selected for blood collection, resulting in a total of 1089 ( $53 \%$ females) blood samples for the subsequent clinical biochemistry assays. Eighty-three per cent of the total sample ( $n$ 3528) responded to a question concerning breakfast consumption, resulting in a final sample of 2929 adolescents ( $53 \%$ females), for whom blood analyses were available for 925 (53\% females). The response frequencies to the breakfast question differed between centres, ranging from 60\% (in Pecs) to 98\% (in Lille and Vienna). Age and BMI were similar between responders and non-responders to the breakfast question $(P>0 \cdot 1)$.

After receiving complete information about the aims and methods of the study, all adolescents and their parents or guardians signed an informed written consent. All participants met the general HELENA inclusion criteria: they were not participating simultaneously in another clinical trial and had not had an acute infection less than 1 week before the study ${ }^{(19)}$. The study was performed following the ethical guidelines of the Declaration of Helsinki 1961 (revision of Edinburgh 2000), Good Clinical Practice and the legislation concerning clinical research in human subjects in each of the participating countries. The protocol was approved by the corresponding local Human Research Review Committees of the centres involved ${ }^{(19,20)}$.

\section{Breakfast assessment}

Adolescents reported their breakfast habits by responding to the following statement: 'I often skip breakfast'. There were seven possible answers ranging from strongly disagree (1) to strongly agree (7). Adolescents were categorized into three groups: (i) 'consumers' (answered ' 1 ' or '2'); (ii) 'occasional consumers' (answered ' 3 ', ' 4 ' or ' 5 '); and (iii) 'skippers' (answered ' 6 ' or ' '7'). The term 'breakfast' was left open to interpretation by the adolescents themselves.

\section{Pbysical examination}

Weight and height were measured following standard procedures ${ }^{(21)}$, and BMI $\left(\mathrm{kg} / \mathrm{m}^{2}\right)$ was calculated as body mass (in kilograms) divided by the square of height (in metres). Adolescents were classified according to the international BMI cut-off values as non-overweight or overweight/obese ${ }^{(22)}$. Waist circumference was used as a surrogate of central body fat, and was measured to the nearest $0.1 \mathrm{~cm}$ in triplicate at the midpoint between the superior iliac spine and the costal edge in the midaxillary line with an anthropometric non-elastic tape (Seca 200; Belgium). Skinfold thickness was measured to the nearest $0.2 \mathrm{~mm}$ in triplicate on the left side at the following sites: biceps, triceps, subscapular, suprailiac, thigh and medial calf, with a Holtain calliper (Crymych, UK) ${ }^{(21)}$. The same trained investigators made all measurements and the inter-rater reliability was greater than $95 \%$.

\section{Assessment of pubertal status}

Overweight/obese adolescents tend to mature earlier than non-overweight ${ }^{(23)}$. Pubertal stage was therefore recorded and adjusted for in the analysis. A trained researcher of the same sex as the child assessed the 
developmental stage according to the scale proposed by Tanner and Whitehouse ${ }^{(24)}$, as described elsewhere ${ }^{(25)}$.

\section{Cardiorespiratory fitness}

Cardiorespiratory fitness was assessed by means of the $20 \mathrm{~m}$ shuttle run test ${ }^{(26)}$. Participants were required to run between two lines $20 \mathrm{~m}$ apart, while keeping pace with audio signals emitted from a pre-recorded compact disk. The initial speed was $8.5 \mathrm{~km} / \mathrm{h}$, which was increased by $0.5 \mathrm{~km} / \mathrm{h}$ each minute ( $1 \mathrm{~min}$ equals one stage). All measurements were carried out under standardized conditions in an indoor gymnasium, during ordinary classes in physical education and simultaneously by ten to twenty adolescents. The participants were encouraged to keep running as long as possible. The last completed stage or half-stage at which the participant dropped out was scored. Cardiorespiratory fitness (i.e. $\mathrm{V}_{\mathrm{O} 2 \max }$ in $\mathrm{ml} / \mathrm{kg}$ per min) was estimated from the last half-stage completed, sex, age, weight and height ${ }^{(27)}$. All participants received comprehensive instructions about the test. This test has shown to be valid ${ }^{(28)}$, reliable ${ }^{(29)}$ and feasible for use in populationbased studies and in the school setting ${ }^{(30)}$.

\section{Physical activity}

We measured physical activity with accelerometry over $7 \mathrm{~d}$ (Actigraph ${ }^{\mathrm{TM}}$ GT1M; Pensacola, FL, USA) and expressed it as total counts $/ \mathrm{min}^{(31)}$.

\section{Blood pressure}

Blood pressure was measured (in the morning) with an automatic oscillometric device (OMRON M6; Omron Healthcare Europe). The adolescent first sat quietly for $5 \mathrm{~min}$, with his/her back supported, feet on the floor and right arm supported with the cubital fossa at heart level. Two recordings of systolic and diastolic measurements (in $\mathrm{mmHg}$ ), $5 \mathrm{~min}$ apart, were made and the lowest value of the two recordings was retained ${ }^{(25)}$.

\section{Blood analysis}

A detailed description of blood sampling and procedures has been published elsewhere ${ }^{(32)}$. Blood samples were drawn after an overnight fast of $10 \mathrm{~h}$. Serum TAG, total cholesterol (TC), HDL cholesterol (HDL-C), LDL cholesterol (LDL-C) and glucose were measured, in single, on the Dimension RxL clinical chemistry system (Dade Behring, Schwalbach, Germany) with enzymatic methods following the manufacturer's reagents and instructions. The intra- and inter-assay CV for all parameters was $<4 \%$. Insulin concentrations were measured by a solid-phase two-site chemiluminescent immunometric assay with an Immulite 2000 analyser (DPC Biermann GmbH, Bad Nauheim, Germany) using the manufacturer's reagents and instructions. The sensitivity of the insulin assay was $2 \mathrm{mU} / \mathrm{l}$. The inter-assay $\mathrm{CV}$ was $5 \cdot 2 \%$. The homeostasis model assessment-insulin resistance index (HOMA-IR) was calculated as [fasting insulin $(\mathrm{mU} / \mathrm{l}) \times$ fasting glucose $(\mathrm{mg} / \mathrm{dl}) \times 0 \cdot 0555 \mathrm{l} / 22 \cdot 5^{(33)}$. Five adolescents with an insulin value $>70 \mathrm{mU} / 1$ were excluded from the analysis of insulin and HOMA-IR.

\section{Sociodemographic status}

Parents' education level was assessed via questionnaire by the adolescent and was categorized as elementary, lower secondary, higher secondary and university level education. We obtained information about family structure through the aforementioned questionnaire. Family structure was defined as 'traditional family' when the adolescent was living at home with two parents (parents and/or stepparents) or 'single/shared-care' when the adolescent was living in a single-parent family or had 'shared care' between parents. Those living in other family structures (e.g. in a foster home or with grandparents) were categorized into the 'single/shared-care' family structure.

\section{Statistical analysis}

Associations between sex and breakfast consumption categories (consumer, occasional consumer and skipper) were assessed by the $\chi^{2}$ test. We compared mean levels of CVD risk factors across breakfast consumption categories using one-way analysis of covariance with breakfast consumption category as the fixed factor, CVD risk factors as dependent variables and age, centre (random variable), mother's and father's education and family structure entered as covariates. Analyses including waist circumference were additionally adjusted for height. All analyses were performed in males and females separately. Variables with skewed distribution (i.e. the sum of six skinfolds, $\mathrm{V}_{\mathrm{O} \text { max }}$, TAG, TC:HDL-C and HOMA-IR) were logarithmically transformed to obtain a more symmetric distribution.

To study the interaction between breakfast consumption and weight status (i.e. non-overweight and overweight/ obese) on CVD risk factors, we performed a two-way analysis of covariance (with breakfast consumption and weight status as fixed factors) adjusting for the covariates mentioned above. A possible breakfast consumption/weight status interaction effect on CVD risk factors was studied by inserting the product term (breakfast consumption $X$ weight status) into the model. All analyses were performed using the SPSS for Windows statistical software package version $16 \cdot 0$ (SPSS Inc., Chicago, IL, USA), and the level of significance was set at $5 \%$.

\section{Results}

The percentage of breakfast consumers was significantly higher in males than in females, in both the total study sample as well as in the subgroup with blood analysis (Table $1, P<0 \cdot 001$ ). In the whole study sample, the percentage of breakfast consumers was significantly higher in females from northern/central Europe than in females from southern Europe (Table 2, $P<0 \cdot 01$ ). In contrast, among 
Table 1 Breakfast consumption, weight status, mother's/father's education level and family structure by sex: adolescents ( $n$ 2929), aged 12-17 years, from ten European cities participating in the HELENA (Healthy Lifestyle in Europe by Nutrition in Adolescence) Study

\begin{tabular}{|c|c|c|c|c|c|c|c|c|c|c|}
\hline & \multicolumn{5}{|c|}{ Total sample } & \multicolumn{5}{|c|}{ Sub-sample* } \\
\hline & \multicolumn{2}{|c|}{ Males } & \multicolumn{2}{|c|}{ Females } & \multirow[b]{2}{*}{$P+$} & \multicolumn{2}{|l|}{ Males } & \multicolumn{2}{|c|}{ Females } & \multirow[b]{2}{*}{$P+$} \\
\hline & Frequency & $\%$ & Frequency & $\%$ & & Frequency & $\%$ & Frequency & $\%$ & \\
\hline \multicolumn{11}{|l|}{ Age } \\
\hline$<15$ years & 760 & 56 & 924 & 59 & 0.038 & 252 & 58 & 309 & 63 & $0 \cdot 152$ \\
\hline$\geq 15$ years & 610 & 44 & 635 & 41 & & 181 & 42 & 183 & 37 & \\
\hline \multicolumn{11}{|l|}{ Breakfast consumption } \\
\hline Consumer & 694 & 51 & 699 & 45 & $<0.001$ & 244 & 56 & 213 & 43 & $<0.001$ \\
\hline Occasional consumer & 344 & 25 & 342 & 22 & & 80 & 18 & 109 & 22 & \\
\hline Skipper & 332 & 24 & 518 & 33 & & 109 & 25 & 170 & 35 & \\
\hline \multicolumn{11}{|l|}{ BMI $\left(\mathrm{kg} / \mathrm{m}^{2}\right)$} \\
\hline Non-overweight & 1007 & 74 & 1243 & 80 & $<0.001$ & 322 & 75 & 396 & 81 & 0.047 \\
\hline Overweight & 260 & 19 & 253 & 16 & & 78 & 18 & 76 & 15 & \\
\hline Obese & 103 & 7 & 63 & 4 & & 33 & 7 & 20 & 4 & \\
\hline \multicolumn{11}{|l|}{ Mother's education } \\
\hline Elementary & 101 & 8 & 111 & 7 & $0 \cdot 768$ & 33 & 8 & 41 & 9 & 0.550 \\
\hline Lower secondary & 328 & 25 & 367 & 25 & & 104 & 26 & 116 & 25 & \\
\hline Higher secondary & 405 & 31 & 497 & 33 & & 127 & 32 & 168 & 36 & \\
\hline University & 453 & 35 & 518 & 35 & & 139 & 34 & 146 & 31 & \\
\hline \multicolumn{11}{|l|}{ Father's education } \\
\hline Elementary & 83 & 7 & 111 & 9 & 0.643 & 31 & 8 & 32 & 7 & 0.885 \\
\hline Lower secondary & 358 & 28 & 409 & 29 & & 108 & 28 & 132 & 29 & \\
\hline Higher secondary & 369 & 29 & 403 & 28 & & 119 & 30 & 145 & 32 & \\
\hline University & 454 & 36 & 504 & 35 & & 134 & 34 & 149 & 33 & \\
\hline \multicolumn{11}{|l|}{ Family structure } \\
\hline Traditional & 1035 & 78 & 1191 & 79 & 0.383 & 335 & 79 & 393 & 83 & 0.225 \\
\hline Single/shared-care & 291 & 22 & 309 & 21 & & 87 & 21 & 83 & 17 & \\
\hline
\end{tabular}

*Sub-sample with blood analysis.

$+P$ value from $\chi^{2}$ test; $P<0.05$ indicates statistical significance.

Table 2 Breakfast consumption by sex and European region: adolescents ( $n$ 2929), aged $12-17$ years, from ten European cities participating in the HELENA (Healthy Lifestyle in Europe by Nutrition in Adolescence) Study

\begin{tabular}{|c|c|c|c|c|c|c|c|c|c|c|}
\hline & \multicolumn{5}{|c|}{ Total sample } & \multicolumn{5}{|c|}{ Sub-sample* } \\
\hline & \multicolumn{2}{|c|}{ Southern } & \multicolumn{2}{|c|}{ Northern/central } & \multirow[b]{2}{*}{$P+$} & \multicolumn{2}{|c|}{ Southern } & \multicolumn{2}{|c|}{ Northern/central } & \multirow[b]{2}{*}{$P+$} \\
\hline & Frequency & $\%$ & Frequency & $\%$ & & Frequency & $\%$ & Frequency & $\%$ & \\
\hline \multicolumn{11}{|l|}{ Males } \\
\hline Consumer & 289 & 58 & 504 & 58 & $<0.001$ & 95 & 58 & 169 & 63 & 0.056 \\
\hline Occasional consumer & 65 & 13 & 60 & 7 & & 16 & 10 & 11 & 4 & \\
\hline Skipper & 146 & 29 & 306 & 35 & & 52 & 32 & 90 & 33 & \\
\hline \multicolumn{11}{|l|}{ Female } \\
\hline Consumer & 271 & 45 & 521 & 55 & $<0.01$ & 84 & 42 & 152 & 52 & $0 \cdot 106$ \\
\hline Occasional consumer & 51 & 8 & 59 & 6 & & 16 & 8 & 21 & 7 & \\
\hline Skipper & 283 & 47 & 374 & 39 & & 99 & 50 & 120 & 41 & \\
\hline
\end{tabular}

${ }^{*}$ Sub-sample with blood analysis

$+P$ value from $\chi^{2}$ test; $P<0.05$ indicates statistical significance.

males from northern/central Europe the percentage of breakfast skippers was significantly higher compared with those from southern Europe (Table 2, $P<0 \cdot 001$ ). No regional differences were seen in the percentage of breakfast consumers/skippers among the subgroup of adolescents with blood analysis. No association was observed between sex and either maternal/paternal education level or family structure (Table 1). Table 3 shows the mean age and values of the studied CVD risk factors by breakfast consumption category and sex. In males, significant differences across breakfast consumption category were seen for age, BMI, skinfold thickness, waist circumference, systolic and diastolic blood pressures (all $P<0 \cdot 001$ ), as well as for TC:HDL-C, LDL-C:HDL-C, glucose, insulin and HOMA-IR (all $P<0 \cdot 01)$ and LDL-C $(P<0 \cdot 05)$. No association was observed between breakfast and TAG, TC or HDL-C (Table 3). In females, significant differences across breakfast consumption category were seen for skinfold thickness $(P<0 \cdot 01) \mathrm{BMI}$, insulin and HOMA-IR (all $P<0 \cdot 05$ ), whereas no association was observed between breakfast consumption category and age or the other studied CVD risk factors. Cardiorespiratory fitness differed significantly by breakfast consumption category in both males and females (both $P<0 \cdot 001$ ). 
Table 3 CVD risk factors by breakfast consumption and sex: adolescents ( $n$ 2929), aged $12-17$ years, from ten European cities participating in the HELENA (Healthy Lifestyle in Europe by Nutrition in Adolescence) Study

\begin{tabular}{|c|c|c|c|c|c|c|c|c|}
\hline & \multicolumn{4}{|c|}{ Males $(n 1370)$} & \multicolumn{4}{|c|}{ Females ( $n$ 1559) } \\
\hline & Mean & SE & $P^{*}$ & $R^{2}$ & Mean & SE & $P^{*}$ & $R^{2}$ \\
\hline \multicolumn{9}{|l|}{ Age (years) } \\
\hline Consumer & $14 \cdot 9$ & $0 \cdot 1$ & \multirow{3}{*}{0.001} & \multirow{3}{*}{$0 \cdot 046$} & $14 \cdot 9$ & $0 \cdot 1$ & \multirow{3}{*}{$0 \cdot 198$} & \multirow{3}{*}{0.035} \\
\hline Occasional consumer & $15 \cdot 0$ & $0 \cdot 1$ & & & $14 \cdot 8$ & $0 \cdot 1$ & & \\
\hline Skipper & $15 \cdot 2$ & $0 \cdot 1$ & & & $15 \cdot 0$ & $0 \cdot 1$ & & \\
\hline \multicolumn{9}{|l|}{ BMI $\left(\mathrm{kg} / \mathrm{m}^{2}\right)$} \\
\hline Consumer & $20 \cdot 9$ & 0.2 & \multirow{3}{*}{$<0.001$} & \multirow{3}{*}{0.072} & $21 \cdot 2$ & $0 \cdot 1$ & \multirow{3}{*}{$0 \cdot 015$} & \\
\hline Occasional consumer & $21 \cdot 8$ & 0.2 & & & $21 \cdot 4$ & 0.2 & & 0.049 \\
\hline Skipper & $22 \cdot 6$ & 0.2 & & & $21 \cdot 8$ & 0.2 & & \\
\hline Sum of six skinfolds ( $\mathrm{mm}$ & & & & & & & & \\
\hline Consumer & $70 \cdot 4$ & 1.5 & & & $100 \cdot 6$ & $1 \cdot 4$ & & \\
\hline Occasional consumer & $77 \cdot 6$ & $2 \cdot 1$ & $<0.001$ & 0.050 & $101 \cdot 6$ & $2 \cdot 1$ & 0.003 & 0.019 \\
\hline Skipper & $80 \cdot 6$ & $2 \cdot 2$ & & & $107 \cdot 0$ & $1 \cdot 7$ & & \\
\hline WC (cm) & & & & & & & & \\
\hline Consumer & $73 \cdot 3$ & 0.4 & & & $70 \cdot 2$ & 0.3 & & \\
\hline Occasional consumer & $75 \cdot 0$ & 0.5 & $<0.001$ & 0.129 & $70 \cdot 5$ & 0.5 & 0.083 & 0.068 \\
\hline Skipper & $76 \cdot 5$ & 0.5 & & & $71 \cdot 3$ & 0.4 & & \\
\hline $\mathrm{V}_{\text {O2max }}(\mathrm{ml} / \mathrm{kg}$ per min $) \dagger$ & & & & & & & & \\
\hline Consumer & $52 \cdot 4$ & $0 \cdot 4$ & & & $36 \cdot 8$ & 0.3 & & \\
\hline Occasional consumer & $50 \cdot 1$ & 0.5 & $<0.001$ & 0.071 & $36 \cdot 3$ & 0.4 & $<0.001$ & 0.096 \\
\hline Skipper & $48 \cdot 5$ & 0.5 & & & $34 \cdot 9$ & 0.3 & & \\
\hline $\mathrm{SBP}(\mathrm{mmHg})$ & & & & & & & & \\
\hline Consumer & 124 & 0.5 & & & 116 & 0.5 & & \\
\hline Occasional consumer & 124 & 0.8 & $<0.001$ & $0 \cdot 112$ & 116 & 0.7 & 0.822 & 0.036 \\
\hline Skipper & 129 & $0 \cdot 8$ & & & 116 & 0.5 & & \\
\hline $\mathrm{DBP}(\mathrm{mmHg})$ & & & & & & & & \\
\hline Consumer & 67 & 0.4 & & & 68 & 0.4 & & \\
\hline Occasional consumer & 67 & 0.5 & $<0.001$ & 0.056 & 68 & 0.5 & 0.636 & 0.011 \\
\hline Skipper & 70 & 0.5 & & & 69 & 0.4 & & \\
\hline Blood markers & & & & & & & & \\
\hline TAG (mg/dl)† & & & & & & & & \\
\hline Consumer & $64 \cdot 2$ & $2 \cdot 1$ & & & $76 \cdot 1$ & $2 \cdot 9$ & & \\
\hline Occasional consumer & $62 \cdot 2$ & 3.9 & 0.138 & 0.024 & $71 \cdot 6$ & $4 \cdot 2$ & 0.835 & 0.001 \\
\hline Skipper & $70 \cdot 7$ & $3 \cdot 2$ & & & $71 \cdot 0$ & $3 \cdot 2$ & & \\
\hline $\mathrm{TC}(\mathrm{mg} / \mathrm{dl})$ & & & & & & & & \\
\hline Consumer & $152 \cdot 5$ & $1 \cdot 8$ & & & $168 \cdot 3$ & $2 \cdot 1$ & & \\
\hline Occasional consumer & $154 \cdot 2$ & $3 \cdot 3$ & $0 \cdot 216$ & $0 \cdot 018$ & $166 \cdot 5$ & $3 \cdot 0$ & 0.889 & 0.005 \\
\hline Skipper & $158 \cdot 2$ & $2 \cdot 7$ & & & $167 \cdot 9$ & $2 \cdot 3$ & & \\
\hline $\mathrm{HDL}-\mathrm{C}(\mathrm{mg} / \mathrm{dl})$ & & & & & & & & \\
\hline Consumer & $53 \cdot 8$ & 0.7 & & & $57 \cdot 9$ & 0.8 & & \\
\hline Occasional consumer & $54 \cdot 0$ & $1 \cdot 2$ & 0.082 & 0.036 & $57 \cdot 2$ & $1 \cdot 2$ & 0.797 & 0.007 \\
\hline Skipper & $51 \cdot 2$ & $1 \cdot 0$ & & & $57 \cdot 2$ & 0.9 & & \\
\hline LDL-C (mg/dl) & & & & & & & & \\
\hline Consumer & $88 \cdot 7$ & $1 \cdot 7$ & & & $97 \cdot 9$ & $1 \cdot 8$ & & \\
\hline Occasional consumer & $89 \cdot 2$ & $3 \cdot 1$ & 0.030 & $0 \cdot 019$ & $96 \cdot 9$ & $2 \cdot 7$ & 0.923 & 0.007 \\
\hline Skipper & $96 \cdot 6$ & $2 \cdot 5$ & & & $98 \cdot 2$ & $2 \cdot 1$ & & \\
\hline TC:HDL-C† & & & & & & & & \\
\hline Consumer & $2 \cdot 91$ & 0.05 & & & $2 \cdot 96$ & 0.04 & & \\
\hline Occasional consumer & $2 \cdot 94$ & 0.09 & 0.007 & 0.015 & $2 \cdot 98$ & 0.06 & 0.842 & 0.005 \\
\hline Skipper & $3 \cdot 19$ & 0.07 & & & 3.02 & 0.05 & & \\
\hline LDL-C:HDL-C & & & & & & & & \\
\hline Consumer & $1 \cdot 72$ & 0.05 & & & $1 \cdot 74$ & 0.04 & & \\
\hline Occasional consumer & $1 \cdot 73$ & 0.08 & 0.008 & 0.016 & $1 \cdot 77$ & 0.06 & 0.686 & 0.004 \\
\hline Skipper & 1.98 & 0.07 & & & $1 \cdot 79$ & 0.05 & & \\
\hline Insulin $(\mu \mathrm{IU} / \mathrm{ml})$ & & & & & & & & \\
\hline Consumer & $8 \cdot 3$ & 0.4 & & & $9 \cdot 3$ & 0.4 & & \\
\hline Occasional consumer & $11 \cdot 1$ & 0.8 & 0.001 & 0.048 & $9 \cdot 2$ & 0.5 & 0.025 & 0.093 \\
\hline Skipper & $10 \cdot 6$ & 0.6 & & & $10 \cdot \overline{6}$ & 0.4 & & \\
\hline Glucose (mg/dl) & & & & & & & & \\
\hline Consumer & $91 \cdot 6$ & 0.5 & & & $88 \cdot 3$ & 0.5 & & \\
\hline Occasional consumer & $92 \cdot 2$ & 0.9 & 0.004 & 0.012 & $89 \cdot 1$ & 0.7 & $0 \cdot 182$ & 0.075 \\
\hline Skipper & $94 \cdot 6$ & $0 \cdot 8$ & & & $89 \cdot 6$ & 0.5 & & \\
\hline HOMA-IRt & & & & & & & & \\
\hline Consumer & 1.9 & $0 \cdot 1$ & & & $2 \cdot 0$ & $0 \cdot 1$ & & \\
\hline Occasional consumer & $2 \cdot 5$ & 0.2 & 0.001 & 0.050 & $2 \cdot 0$ & $0 \cdot 1$ & 0.038 & $0 \cdot 103$ \\
\hline Skipper & $2 \cdot 5$ & $0 \cdot 1$ & & & $2 \cdot 4$ & $0 \cdot 1$ & & \\
\hline
\end{tabular}

WC, waist circumference; $\mathrm{V}_{\mathrm{O} \text { max }}$, cardiorespiratory fitness; SBP, systolic blood pressure; DBP, diastolic blood pressure; TC, total cholesterol; HDL-C, HDL cholesterol; LDL-C, LDL cholesterol; HOMA-IR, homeostasis model assessment-insulin resistance index.

All analyses were adjusted for centre (random variable), age, mother's education, father's education and family structure.

${ }^{*} P$ value from one-way analysis of covariance; $P<0.05$ indicates statistical significance.

†Analysis was performed on log-transformed data, but non-transformed data are presented as mean and SE. 
Table 4 CVD risk factors by breakfast consumption, sex and weight status: adolescents ( $n$ 2929), aged $12-17$ years, from ten European cities participating in the HELENA (Healthy Lifestyle in Europe by Nutrition in Adolescence) Study

\begin{tabular}{|c|c|c|c|c|c|c|c|c|c|c|c|c|c|c|c|c|}
\hline & \multicolumn{8}{|c|}{ Males } & \multicolumn{8}{|c|}{ Females } \\
\hline & \multicolumn{2}{|c|}{$\begin{array}{l}\text { Non-overweight } \\
(n \text { 1007) }\end{array}$} & \multicolumn{2}{|c|}{$\begin{array}{l}\text { Overweight/obese } \\
\quad(n \text { 363) }\end{array}$} & \multirow[b]{2}{*}{$P^{\star}$} & \multirow[b]{2}{*}{$P+$} & \multirow[b]{2}{*}{$P \ddagger$} & \multirow[b]{2}{*}{$R^{2}$} & \multicolumn{2}{|c|}{$\begin{array}{l}\text { Non-overweight } \\
\quad(n \text { 1243) }\end{array}$} & \multicolumn{2}{|c|}{$\begin{array}{l}\text { Overweight/obese } \\
\qquad(n 316)\end{array}$} & \multirow[b]{2}{*}{$P^{*}$} & \multirow[b]{2}{*}{$P+$} & \multirow[b]{2}{*}{$P \neq$} & \multirow[b]{2}{*}{$R^{2}$} \\
\hline & Mean & SE & Mean & SE & & & & & Mean & SE & Mean & SE & & & & \\
\hline \multicolumn{17}{|l|}{$\overline{V_{\text {O2max }}(\mathrm{ml} / \mathrm{kg} \text { per } \min ) \S}$} \\
\hline Consumer & $54 \cdot 6$ & 0.3 & $43 \cdot 6$ & 0.7 & \multirow{3}{*}{$<0.001$} & \multirow{3}{*}{0.001} & \multirow{3}{*}{0.651} & \multirow{3}{*}{$0 \cdot 361$} & $37 \cdot 7$ & 0.3 & $32 \cdot 3$ & 0.7 & & & & \\
\hline Occasional consumer & $53 \cdot 0$ & 0.5 & $41 \cdot 5$ & 0.9 & & & & & $36 \cdot 5$ & 0.4 & $33 \cdot 6$ & 0.8 & $<0.001$ & 0.003 & 0.083 & $0 \cdot 182$ \\
\hline Skipper & 51.8 & 0.6 & $41 \cdot 7$ & 0.8 & & & & & $35 \cdot 4$ & 0.4 & $31 \cdot 6$ & $0 \cdot 7$ & & & & \\
\hline $\mathrm{SBP}(\mathrm{mmHg})$ & & & & & & & & & & & & & & & & \\
\hline Consumer & 122 & 0.6 & 131 & $1 \cdot 1$ & & & & & 115 & 0.5 & 122 & $1 \cdot 1$ & & & & \\
\hline Occasional consumer & 122 & 0.9 & 130 & $1 \cdot 4$ & $<0.001$ & $<0.001$ & $0 \cdot 211$ & $0 \cdot 186$ & 115 & 0.7 & 123 & $1 \cdot 4$ & $<0.001$ & $0 \cdot 861$ & 0.834 & $0 \cdot 100$ \\
\hline Skipper & 125 & 0.9 & 136 & $1 \cdot 3$ & & & & & 115 & 0.6 & 122 & $1 \cdot 1$ & & & & \\
\hline $\mathrm{DBP}(\mathrm{mmHg})$ & & & & & & & & & & & & & & & & \\
\hline Consumer & 66 & 0.4 & 69 & 0.8 & & & & & 68 & 0.4 & 72 & 0.9 & & & & \\
\hline Occasional consumer & 66 & 0.6 & 70 & $1 \cdot 0$ & $<0.001$ & $<0.001$ & 0.664 & 0.080 & 67 & 0.6 & 73 & $1 \cdot 1$ & $<0.001$ & 0.884 & 0.300 & 0.052 \\
\hline Skipper & 69 & 0.6 & 72 & 0.8 & & & & & 68 & 0.5 & 72 & 0.9 & & & & \\
\hline Blood parameters & $(n$ & & & & & & & & $(n$ & & & & & & & \\
\hline TAG $(\mathrm{mg} / \mathrm{dl}) \S$ & & & & & & & & & & & & & & & & \\
\hline Consumer & $60 \cdot 5$ & $2 \cdot 4$ & $76 \cdot 5$ & $4 \cdot 4$ & & & & & $71 \cdot 4$ & $3 \cdot 1$ & $99 \cdot 2$ & $7 \cdot 0$ & & & & \\
\hline Occasional consumer & $59 \cdot 2$ & $4 \cdot 3$ & $74 \cdot 2$ & $8 \cdot 6$ & $<0.001$ & 0.410 & 0.924 & 0.066 & $70 \cdot 9$ & $4 \cdot 5$ & $75 \cdot 1$ & $10 \cdot 1$ & 0.006 & 0.393 & 0.465 & 0.017 \\
\hline Skipper & $65 \cdot 3$ & $3 \cdot 9$ & $79 \cdot 8$ & $5 \cdot 0$ & & & & & $68 \cdot 2$ & 3.6 & $80 \cdot 0$ & $6 \cdot 4$ & & & & \\
\hline $\mathrm{TC}(\mathrm{mg} / \mathrm{dl})$ & & & & & & & & & & & & & & & & \\
\hline Consumer & $153 \cdot 9$ & $2 \cdot 0$ & $147 \cdot 9$ & 3.8 & & & & & $168 \cdot 3$ & $2 \cdot 3$ & $168 \cdot 1$ & $5 \cdot 2$ & & & & \\
\hline Occasional consumer & $151 \cdot 5$ & $3 \cdot 6$ & $165 \cdot 3$ & $7 \cdot 4$ & $0 \cdot 109$ & 0.034 & 0.025 & 0.037 & $165 \cdot 7$ & 3.3 & $170 \cdot 9$ & $7 \cdot 5$ & 0.413 & 0.980 & 0.812 & 0.010 \\
\hline Skipper & 154.5 & $3 \cdot 3$ & $164 \cdot 0$ & $4 \cdot 3$ & & & & & $166 \cdot 9$ & $2 \cdot 7$ & $171 \cdot 0$ & $4 \cdot 7$ & & & & \\
\hline HDL-C (mg/dl) & & & & & & & & & & & & & & & & \\
\hline Consumer & $55 \cdot 5$ & 0.7 & $47 \cdot 9$ & $1 \cdot 4$ & & & & & $59 \cdot 1$ & 0.9 & $52 \cdot 0$ & $2 \cdot 0$ & & & & \\
\hline Occasional consumer & $54 \cdot 7$ & $1 \cdot 3$ & $50 \cdot 8$ & $2 \cdot 7$ & $<0.001$ & 0.377 & 0.526 & $0 \cdot 108$ & $57 \cdot 6$ & $1 \cdot 3$ & $55 \cdot 2$ & $2 \cdot 8$ & 0.002 & 0.990 & 0.413 & 0.022 \\
\hline Skipper & $53 \cdot 3$ & $1 \cdot 2$ & $47 \cdot 5$ & $1 \cdot 6$ & & & & & $58 \cdot 2$ & $1 \cdot 0$ & $53 \cdot \overline{9}$ & $1 \cdot 8$ & & & & \\
\hline LDL-C (mg/dl) & & & & & & & & & & & & & & & & \\
\hline Consumer & $88 \cdot 3$ & $1 \cdot 8$ & $90 \cdot 0$ & 3.5 & & & & & $97 \cdot 1$ & $2 \cdot 0$ & $101 \cdot 6$ & $4 \cdot 5$ & & & & \\
\hline Occasional consumer & $85 \cdot 9$ & $3 \cdot 4$ & $102 \cdot 7$ & $6 \cdot 8$ & 0.001 & 0.015 & 0.049 & 0.048 & $95 \cdot 6$ & $2 \cdot 9$ & $103 \cdot 3$ & $6 \cdot 6$ & 0.057 & 0.982 & 0.914 & 0.009 \\
\hline Skipper & $90 \cdot 6$ & $3 \cdot 1$ & $106 \cdot 2$ & $4 \cdot 0$ & & & & & $96 \cdot 5$ & $2 \cdot 4$ & 103.5 & $4 \cdot 2$ & & & & \\
\hline TC:HDL-C\$ & & & & & & & & & & & & & & & & \\
\hline Consumer & $2 \cdot 85$ & 0.05 & $3 \cdot 14$ & $0 \cdot 10$ & & & & & $2 \cdot 89$ & 0.05 & $3 \cdot 31$ & $0 \cdot 11$ & & & & \\
\hline Occasional consumer & $2 \cdot 83$ & $0 \cdot 10$ & $3 \cdot 36$ & 0.19 & $<0.001$ & 0.024 & 0.414 & 0.078 & $2 \cdot 94$ & 0.07 & $3 \cdot 21$ & $0 \cdot 16$ & $<0.001$ & 0.962 & 0.775 & 0.039 \\
\hline Skipper & 3.00 & 0.09 & 3.49 & 0.11 & & & & & 2.95 & 0.06 & 3.27 & $0 \cdot 10$ & & & & \\
\hline LDL-C:HDL-C & & & & & & & & & & & & & & & & \\
\hline Consumer & 1.66 & 0.05 & 1.92 & 0.09 & & & & & 1.68 & 0.04 & $2 \cdot 01$ & $0 \cdot 10$ & & & & \\
\hline Occasional consumer & 1.63 & 0.09 & $2 \cdot 12$ & $0 \cdot 18$ & $<0.001$ & 0.023 & 0.354 & 0.067 & $1 \cdot 72$ & 0.06 & 1.99 & $0 \cdot 15$ & $<0.001$ & 0.979 & 0.909 & 0.036 \\
\hline Skipper & $1 \cdot 79$ & 0.08 & $2 \cdot 27$ & 0.11 & & & & & 1.73 & 0.05 & 1.99 & 0.09 & & & & \\
\hline Insulin $(\mu \mathrm{IU} / \mathrm{ml})$ & & & & & & & & & & & & & & & & \\
\hline Consumer & $7 \cdot 4$ & 0.4 & $11 \cdot 5$ & 0.8 & & & & & $8 \cdot 6$ & 0.4 & $12 \cdot 9$ & 0.8 & & & & \\
\hline Occasional consumer & $10 \cdot 7$ & 0.8 & $12 \cdot 5$ & $1 \cdot 6$ & $<0.001$ & 0.039 & 0.489 & $0 \cdot 103$ & $9 \cdot 1$ & 0.5 & $9 \cdot 6$ & $1 \cdot 2$ & $<0.001$ & 0.031 & 0.061 & $0 \cdot 160$ \\
\hline Skipper & $9 \cdot 5$ & $0 \cdot 7$ & $12 \cdot 5$ & $1 \cdot 0$ & & & & & $9 \cdot 9$ & 0.4 & $13 \cdot 1$ & $0 . \overline{8}$ & & & & \\
\hline
\end{tabular}




\section{Interactions between breakfast consumption categories and weight status}

Table 4 presents the means of the CVD risk factors by breakfast consumption category, sex and weight status. In male adolescents, an interaction effect on TC and LDL-C was observed between breakfast consumption and weight status. Males who were breakfast consumers and overweight/obese had lower TC and LDL-C compared with the skipper group $(P<0 \cdot 001)$, whereas no association was observed in the non-overweight group. In females, an interaction between breakfast consumption and weight status was observed for glucose. These results persisted after excluding the underweight adolescents from the analysis (data not shown) and when the analyses were additionally adjusted for pubertal status (data not shown). The analyses were repeated after further adjusting for objectively measured physical activity, and the results did not change (data not shown).

\section{Discussion}

The present study, conducted in a relatively large sample of adolescents from nine different European countries, confirmed previous data indicating that adolescents who regularly consume breakfast have lower body fat content. The results also showed that regular breakfast consumption is associated with higher cardiorespiratory fitness in both males and females, and with a healthier cardiovascular profile, especially in males.

The frequency of regular breakfast consumption in our study sample was similar to that reported in other Western populations ${ }^{(3,4)}$. Our study showed that more males than females reported to be regular breakfast consumers. We also observed that older male adolescents were more likely to be breakfast skippers than younger male adolescents. Previous studies from large epidemiological and cross-sectional surveys have observed marked declines in the frequency of breakfast consumption from childhood to adolescence ${ }^{(12,34,35)}$.

In the present study, regular breakfast consumers had lower total adiposity (estimated by BMI or skinfold thickness). Two systematic reviews have examined the association between breakfast consumption and body weight $^{(3,4)}$. Rampersaud et al. concluded that although breakfast eaters consumed more energy daily, they were less likely to be obese; yet they noted that not all studies reported significant relationships between breakfast skipping and overweight/obesity ${ }^{(3)}$. In a recent review Szajewska and Ruszczynski concluded that in European children and adolescents consuming breakfast is associated with a lower BMI and with a reduced risk of becoming overweight or obese ${ }^{(4)}$, which concurs with our results. Our results showed differences in breakfast consumption, according to sex, between the two regions of Europe (southern $v$. northern/central). To the best of 
our knowledge, there are no studies investigating differences in breakfast consumption among adolescents from southern and northern/central Europe. Vereecken et al. have shown differences among countries in Europe: in the southern region daily breakfast consumption ranges from 33\% (Greek girls) to $72 \%$ (Spanish boys) and in the northern/central region it ranges from $42 \%$ (Hungarian girls) to $73 \%$ (Swedish boys) $^{(36)}$. Further research is needed on a regional and/or national level to better understand the breakfast consumption among adolescents living in different parts of Europe.

Breakfast is, for adolescents, one of the most important meals of the day and its consumption is associated with favourable diet quality (i.e. favourable nutrient and energy intakes) and improved food choice ${ }^{(6)}$ and exercise patterns $^{(8)}$. Thus, skipping breakfast has been proposed to influence weight status indirectly by leading to hunger in the morning and resulting in increased snacking and consumption of empty calories ${ }^{(6)}$. On the other hand, it could be the food and nutrient content of breakfast itself that influences body weight ${ }^{(34,37,38)}$. Indeed, several studies have shown that the consumption of high-fibre or wholegrain cereals at breakfast is associated with lower $\mathrm{BMI}^{(34,39,40)}$

We observed that regular breakfast consumption was associated with higher cardiorespiratory fitness in both males and females. These findings confirm the results of a recent report conducted in large sample of schoolchildren $^{(41)}$. Our study showed that boys and girls who never ate breakfast had lower mean cardiorespiratory fitness. A possible link between breakfast consumption and fitness could be due to a clustering of healthy behaviours $^{(8,42,43)}$. Breakfast consumers seem to have a more active lifestyle than breakfast skippers ${ }^{(44)}$. In a previous study ${ }^{(8)}$, the authors showed that breakfast skippers were much more likely to exercise infrequently than regular breakfast consumers.

Regular breakfast consumption was associated with a healthier cardiovascular profile (i.e. waist circumference, blood pressure, TC:HDL-C, LDL-C:HDL-C and insulin resistance) in male adolescents in the present study. Waist circumference is a surrogate measure of abdominal adiposity and is considered an important contributor to metabolic complications in children and adolescents ${ }^{(45)}$. Studies investigating the relationship between breakfast consumption and body fat distribution in adolescents are scarce. Deshmukh-Taskar et al. showed that mean waist circumference was higher in breakfast skippers ${ }^{(37)}$, which concurs with our results. Moreover, a previous study conducted in a well-characterized sample of ninety-three overweight youths (aged 10-17 years) reported that eating breakfast was associated with lower visceral adiposity as measured by dual-energy X-ray absorptiometry ${ }^{(46)}$.

Male breakfast consumers in the present study had lower systolic and diastolic blood pressures regardless of BMI, whereas no association was found in females. Youths who usually consume breakfast are more likely to be frequent consumers of fruit, cereals and milk ${ }^{(47)}$, which, in turn, are central foods in the recommended dietary pattern for lowering blood pressure ${ }^{(48,49)}$. Therefore, the intake of milk products during breakfast supports total daily intakes of milk and $\mathrm{Ca}^{(6,10,50)}$, which have been associated with a lower risk of hypertension in adults $^{(51)}$. In agreement with our findings, a sex-specific association (observed in males only) between breakfast and blood pressure was reported in a previous study performed in Greek adolescents ${ }^{(52)}$.

We did not find any significant effect of breakfast consumption on most of the CVD risk factors, such as blood lipid levels, blood pressure or insulin resistance, in females. Regular breakfast consumption was significantly related to a healthier blood lipid profile in males. Breakfast consumers had lower TC, LDL-C and lower TC:HDL-C and LDL-C:HDL-C ratios. Moreover, our results suggested that consuming breakfast regularly may influence the negative effects of being overweight in males. To our knowledge, there are no previous studies examining the relationship between breakfast consumption and blood metabolic variables. Albertson et al. examined possible sex-related differences in the association between the consumption of ready-to-eat cereals at breakfast and cardiovascular health indicators, showing that ready-toeat cereals were significantly associated with lower blood lipid levels only in males ${ }^{(34)}$. Other studies have also documented a lack of significant associations between food group consumption and cholesterol among females, suggesting a complex association between dietary patterns, blood lipids and sex.

The use of the self-reported statement 'I often skip breakfast' to gauge habitual breakfast consumption could be a limitation of our study. The term 'breakfast consumers' in the literature includes a variety of definitions, such as consuming breakfast every day, every week day, on the dietary survey day, or usual or habitual consumption $^{(3)}$, which makes comparisons difficult. In addition, there is no consensus regarding how to define breakfast consumption. A recent study found that the percentage of breakfast skippers varied greatly according to how breakfast was categorized ${ }^{(53)}$. Furthermore, because of the cross-sectional nature of the study design, no conclusion can be drawn about the directionality and causality of the associations seen between breakfast consumption and CVD risk factors. The large sample of adolescents in the study population and the standardized and harmonized methodology are notable strengths of the present study. In addition, previous studies have predominantly used BMI as a measure of body composition. Recent systematic reviews indicated that skinfold thicknesses and waist circumference are valid makers of total and central fatness in young people $\mathrm{e}^{(28)}$ and are strong predictors of future health status $^{(54)}$. The inclusion of these two surrogates of fatness, and the consistency of the results observed, strengthen our study's conclusions. 


\section{Conclusions}

Our findings in European adolescents confirm previous data indicating that those who regularly consume breakfast have lower body fat. The results also indicate that regular breakfast consumption is associated with higher cardiorespiratory fitness in both males and females, and with a healthier cardiovascular profile, especially in males. Eating breakfast regularly may also negate somewhat the effect of excess adiposity on TC and LDL-C in male adolescents.

\section{Acknowledgements}

The study took place with financial support of the European Community Sixth RTD Framework Programme (Contract FOOD-CT-2005-007034), the Swedish Council for Working Life and Social Research (FAS), the Swedish Heart-Lung Foundation (20090635) and the Spanish Ministry of Health: Maternal, Child Health and Development Network (number RG08/0072). The content of this article reflects the authors' views only and the European Community is not liable for any use that may be made of the information contained herein. None of the authors had a personal or financial conflict of interest. The writing group takes sole responsibility for the content of this article. L.H., I.L. and J.R.R. wrote the manuscript and performed the statistical analysis; L.H., I.L., J.R.R., E.P., C.A.V., C.B., F.G., I.H., Y.M., L.M., K.W., L.A.M. and M.S. contributed to the interpretation and discussion of the results and critically revised the drafted manuscript. The authors thank all the adolescents who took part in the HELENA Study.

\section{References}

1. European Association for the Study of Obesity (2010) Childhood Obesity Task Force: Facts and Statistics. London: EASO; available at http://www.easoobesity.org/task_ forces/childhood_facts_statistics.htm

2. Dubois L, Girard M, Potvin Kent M et al. (2009) Breakfast skipping is associated with differences in meal patterns, macronutrient intakes and overweight among pre-school children. Public Health Nutr 12, 19-28.

3. Rampersaud GC, Pereira MA, Girard BL et al. (2005) Breakfast habits, nutritional status, body weight, and academic performance in children and adolescents. $J$ Am Diet Assoc 105, 743-760.

4. Szajewska H \& Ruszczynski M (2010) Systematic review demonstrating that breakfast consumption influences body weight outcomes in children and adolescents in Europe. Crit Rev Food Sci Nutr 50, 113-119.

5. Merten MJ, Williams AL \& Shriver LH (2009) Breakfast consumption in adolescence and young adulthood: parental presence, community context, and obesity. J Am Diet Assoc 109, 1384-1391.

6. Matthys C, De Henauw S, Bellemans M et al. (2007) Breakfast habits affect overall nutrient profiles in adolescents. Public Health Nutr 10, 413-421.

7. Williams BM, O'Neil CE, Keast DR et al. (2009) Are breakfast consumption patterns associated with weight status and nutrient adequacy in African-American children? Public Health Nutr 12, 489-496.

8. Keski-Rahkonen A, Kaprio J, Rissanen A et al. (2003) Breakfast skipping and health-compromising behaviors in adolescents and adults. Eur J Clin Nutr 57, 842-853.

9. Serra-Majem L, Ribas L, Perez-Rodrigo C et al. (2002) Determinants of nutrient intake among children and adolescents: results from the enKid Study. Ann Nutr Metab 46, Suppl. 1, 31-38.

10. van den Boom A, Serra-Majem L, Ribas L et al. (2006) The contribution of ready-to-eat cereals to daily nutrient intake and breakfast quality in a Mediterranean setting. J Am Coll Nutr 25, 135-143.

11. Viteri FE \& Gonzalez H (2002) Adverse outcomes of poor micronutrient status in childhood and adolescence. Nutr Rev 60, 5 Pt 2, S77-S83.

12. Alexy U, Wicher M \& Kersting M (2010) Breakfast trends in children and adolescents: frequency and quality. Public Health Nutr 13, 1795-1802.

13. Dietz WH (1998) Health consequences of obesity in youth: childhood predictors of adult disease. Pediatrics 101, 518-525.

14. Berenson GS, Srinivasan SR, Wattigney WA et al. (1993) Obesity and cardiovascular risk in children. Ann N Y Acad Sci 699, 93-103.

15. Raitakari OT, Porkka KV, Viikari JS et al. (1994) Clustering of risk factors for coronary heart disease in children and adolescents. The Cardiovascular Risk in Young Finns Study. Acta Paediatr 83, 935-940.

16. Freedman DS, Dietz WH, Srinivasan SR et al. (1999) The relation of overweight to cardiovascular risk factors among children and adolescents: the Bogalusa Heart Study. Pediatrics 103, 1175-1182.

17. Chen W, Srinivasan SR, Li S et al. (2005) Metabolic syndrome variables at low levels in childhood are beneficially associated with adulthood cardiovascular risk: the Bogalusa Heart Study. Diabetes Care 28, 126-131.

18. Chen X \& Wang Y (2008) Tracking of blood pressure from childhood to adulthood: a systematic review and metaregression analysis. Circulation 117, 3171-3180.

19. Moreno LA, De Henauw S, Gonzalez-Gross M et al. (2008) Design and implementation of the Healthy Lifestyle in Europe by Nutrition in Adolescence Cross-Sectional Study. Int J Obes (Lond) 32, Suppl. 5, S4-S11.

20. Beghin L, Castera M, Manios Y et al. (2008) Quality assurance of ethical issues and regulatory aspects relating to good clinical practices in the HELENA Cross-Sectional Study. Int J Obes (Lond) 32, Suppl. 5, S12-S18.

21. Nagy E, Vicente-Rodriguez G, Manios Y et al. (2008) Harmonization process and reliability assessment of anthropometric measurements in a multicenter study in adolescents. Int J Obes (Lond) 32, Suppl. 5, S58-S65.

22. Cole TJ, Bellizzi MC, Flegal KM et al. (2000) Establishing a standard definition for child overweight and obesity worldwide: international survey. Br Med J320, 1240-1243.

23. Garn SM \& Clark DC (1975) Nutrition, growth, development, and maturation: findings from the ten-state nutrition survey of 1968-1970. Pediatrics 56, 306-319.

24. Tanner JM \& Whitehouse RH (1962) Standards for subcutaneous fat in British children. Percentiles for thickness of skinfolds over triceps and below scapula. Br Med J 1, 446-450.

25. Iliescu C, Beghin L, Maes L et al. (2008) Socioeconomic questionnaire and clinical assessment in the HELENA Cross-Sectional Study: methodology. Int J Obes (Lond) 32, Suppl. 5, S19-S25.

26. Leger LA, Mercier D, Gadoury C et al. (1988) The multistage 20 metre shuttle run test for aerobic fitness. J Sports Sci $\mathbf{6}$, 93-101.

27. Ruiz JR, Ramirez-Lechuga J, Ortega FB et al. (2008) Artificial neural network-based equation for estimating $\mathrm{V}_{\mathrm{O} 2 \max }$ from 
the $20 \mathrm{~m}$ shuttle run test in adolescents. Artif Intell Med $\mathbf{4 4}$, 233-245.

28. Castro-Piñero J, Artero EG, España-Romero V et al. (2009) Criterion-related validity of field-based fitness tests in youth: a systematic review. BrJ Sports Med 44, 934-943.

29. Ortega FB, Artero EG, Ruiz JR et al. (2008) Reliability of health-related physical fitness tests in European adolescents. The HELENA Study. Int J Obes (Lond) 32, Suppl. 5, S49-S57.

30. Ruiz JR, Ortega FB, Gutierrez A et al. (2006) Health-related fitness assessment in childhood and adolescence: a European approach based on the AVENA, EYHS and HELENA studies. J Public Health 14, 269-277.

31. Ruiz JR, Ortega FB, Martinez-Gomez D et al. (2011) Objectively measured physical activity and sedentary time in European adolescents: the HELENA study. $A m J$ Epidemiol 174, 173-184.

32. Gonzalez-Gross M, Breidenassel C, Gomez-Martinez S et al. (2008) Sampling and processing of fresh blood samples within a European multicenter nutritional study: evaluation of biomarker stability during transport and storage. Int J Obes (Lond) 32, Suppl. 5, S66-S75.

33. Wallace TM, Levy JC \& Matthews DR (2004) Use and abuse of HOMA modeling. Diabetes Care 27, 1487-1495.

34. Albertson AM, Anderson GH, Crockett SJ et al. (2003) Ready-to-eat cereal consumption: its relationship with BMI and nutrient intake of children aged 4 to 12 years. J Am Diet Assoc 103, 1613-1619.

35. Timlin MT, Pereira MA, Story M et al. (2008) Breakfast eating and weight change in a 5 -year prospective analysis of adolescents: Project EAT (Eating Among Teens). Pediatrics 121, e638-e645.

36. Vereecken CA, Dupuy M, Rasmussen M et al. (2009) Breakfast consumption and its socio-demographic and lifestyle correlates in schoolchildren in 41 countries participating in the HBSC study. Int J Public Health 54, Suppl. 2, 180-190.

37. Deshmukh-Taskar PR, Nicklas TA, O'Neil CE et al. (2010) The relationship of breakfast skipping and type of breakfast consumption with nutrient intake and weight status in children and adolescents: the National Health and Nutrition Examination Survey 1999-2006. J Am Diet Assoc 110, 869-878.

38. Gibson SA \& O'Sullivan KR (1995) Breakfast cereal consumption patterns and nutrient intakes of British schoolchildren. J R Soc Health 115, 366-370.

39. Barton BA, Eldridge AL, Thompson D et al. (2005) The relationship of breakfast and cereal consumption to nutrient intake and body mass index: the National Heart, Lung, and Blood Institute Growth and Health Study. J Am Diet Assoc 105, 1383-1389.

40. Cho S, Dietrich M, Brown CJ et al. (2003) The effect of breakfast type on total daily energy intake and body mass index: results from the Third National Health and Nutrition
Examination Survey (NHANES III). J Am Coll Nutr 22, 296-302.

41. Sandercock GR, Voss C \& Dye L (2010) Associations between habitual school-day breakfast consumption, body mass index, physical activity and cardiorespiratory fitness in English schoolchildren. Eur J Clin Nutr 64, 1086-1092.

42. Aarnio M, Winter T, Kujala U et al. (2002) Associations of health related behaviour, social relationships, and health status with persistent physical activity and inactivity: a study of Finnish adolescent twins. Br J Sports Med 36, 360-364.

43. Cohen B, Evers S, Manske S et al. (2003) Smoking, physical activity and breakfast consumption among secondary school students in a southwestern Ontario community. Can J Public Health 94, 41-44.

44. Corder K, van Sluijs EM, Steele RM et al. (2011) Breakfast consumption and physical activity in British adolescents. Br J Nutr 105, 316-321.

45. Li C, Ford ES, Mokdad AH et al. (2006) Recent trends in waist circumference and waist-height ratio among US children and adolescents. Pediatrics 118, e1390-e1398.

46. Alexander KE, Ventura EE, Spruijt-Metz D et al. (2009) Association of breakfast skipping with visceral fat and insulin indices in overweight Latino youth. Obesity (Silver Spring) 17, 1528-1533.

47. Utter J, Scragg R, Mhurchu CN et al. (2007) At-home breakfast consumption among New Zealand children: associations with body mass index and related nutrition behaviors. J Am Diet Assoc 107, 570-576.

48. Champagne CM (2006) Dietary interventions on blood pressure: the Dietary Approaches to Stop Hypertension (DASH) trials. Nutr Rev 64, 2 Pt 2, S53-S56.

49. Mitka M (2007) DASH dietary plan could benefit many, but few hypertensive patients follow it. J Am Med Assoc 298, 164-165.

50. Ortega RM, Requejo AM, Lopez-Sobaler AM et al. (1998) The importance of breakfast in meeting daily recommended calcium intake in a group of schoolchildren. $J \mathrm{Am}$ Coll Nutr 17, 19-24.

51. Hajjar IM \& Grim CE \& Kotchen TA (2003) Dietary calcium lowers the age-related rise in blood pressure in the United States: the NHANES III survey. J Clin Hypertens (Greenwich) 5, 122-126.

52. Kollias A, Antonodimitrakis P, Grammatikos E et al. (2009) Trends in high blood pressure prevalence in Greek adolescents. J Hum Hypertens 23, 385-390.

53. Dialektakou KD \& Vranas PB (2008) Breakfast skipping and body mass index among adolescents in Greece: whether an association exists depends on how breakfast skipping is defined. J Am Diet Assoc 108, 1517-1525.

54. Ruiz JR, Castro-Piñero J, Artero EG et al. (2009) Predictive validity of health-related fitness in youth: a systematic review. Br J Sports Med 43, 909-923. 